\title{
Pyoderma gangrenosum in patient with rheumatoid arthritis
}

\author{
Barros CE, Concha DC, Tapias SA
}

\begin{abstract}
Introduction: Skin ulcers, especially those whose location is in the lower limbs, involve a wide range of causes and appropriate clinical analysis to be performed. Case Report: We present a case report of a 64-year-old female patient diagnosed with rheumatoid arthritis mutilans, who had suffered for three years ulcerative lesions in the lower limbs. The initial approach included antibiotic management, with a subsequent histopathological diagnosis of pyoderma gangrenosum. She was treated with high doses of corticosteroids and cyclosporine, showing response in facial and knee lesions, however, the left lower limb lesion developed ischemia and subsequent necrosis, requiring suprapatellar amputation. Conclusion: The strong association between systemic diseases of the patient, as well as the differential diagnoses, the difficulty between identification and treatment, make each case of pyoderma gangrenosum a challenge for the health personnel. Underlying pathologies, characteristics of the lesions and biopsy reports should always be considered for an adequate diagnosis.
\end{abstract}

Keywords: Pyoderma gangrenosum, Rheumatoid arthritis, Ulceretive lesion

Barros $\mathrm{CE}^{1}$, Concha DC ${ }^{1}$, Tapias $\mathrm{SA}^{1}$

Affiliation: ${ }^{1}$ Internal Medicine Department, Hospital Universitario Samaritana, Bogota, Colombia.

Corresponding Author: Camilo Ernesto Barros, Internal Medicine Department, Hospital Universitario Samaritana, Bogota, Colombia; Email: camilo_barros@hotmail.com

Received: 26 March 2018

Accepted: 10 May 2018

Published: 31 May 2018

\section{How to cite this article}

Barros CE, Concha DC, Tapias SA. Pyoderma gangrenosum in patient with rheumatoid arthritis. Int J Case Rep Images 2018;9:100919Z01BC2018.

Article ID: 100919Zo1BC2018

$* * * * * * * * *$

doi: 10.5348/100919Z01BC2018CR

\section{INTRODUCTION}

Pyodermagangrenosum is defined as rare, chronic, and destructive skin pathology, which may be in association with systemic diseases, these are ulcerative colitis, Crohn's disease, seronegative arthritis, spondylitis, rheumatoid arthritis, myelogenous leukaemia, hairy cell leukaemia, myelofibrosis and monoclonal gammopathy [1]. It is a neutrophilic dermatosis, which histopathologically has an aseptic infiltration of neutrophils in the epidermis, dermis or subcutaneous tissue [2]. The classic ulcerative lesion is characterized by starting with a sterile pustule or nodule which progresses to necrotic lesions with edematous, violaceous and undermined edges with a mucopurulent base, which progressively extend possibly leading to tendinous and bony exposure [3, 4]. In the year 1916 Brocq used the term 'phagédenismegéométrique' to describe pyoderma gangrenosum, but it was not until 1930 that Brunsting, Goeckerman and O'Leary used the term pyoderma gangrenosum for the first time [1]. Its annual incidence is $3-10$ cases per million people per year, presenting more frequently in the female gender with an age group between $20-50$ years and in association with other diseases by 50 to $70 \%$ [5]. Although the etiology of pyoderma gangrenosum is not clear, it is known that the cause is not infectious and that it has variants in its presentation such as: classic or ulcerous, periostomal, pustular, bullous and vegetative, with characteristic 


\section{EDORIUM Journals}

lesions for each type $[6,7]$. The diagnosis of the disease is clinical since there are no specific serological test or histological markers, criteria such as: rapid progression of the lesion, painful skin ulcer, development of pathergy, associated systemic diseases can be considered [6]. There is no treatment with clinical evidence that is $100 \%$ effective in the treatment of pyoderma gangrenosum. According to the literature, the best results are achieved with the application of various topical medications, such as: hydrocolloid dressings, steroid injection, tacrolimus, in addition to systemic therapy with oral prednisone and cyclosporine in refractory cases [8]. This report presents a case of pyoderma gangrenosum in a patient with rheumatoid arthritis, atypical head and face lesions location, as well as involvement of the right and left lower limbs, with response to different treatment at each location of the lesions.

\section{CASE REPORT}

A 64-year-old female patient admitted to the emergency department, referred from the wound clinic, with a clinical presentation of one year of evolution of the right retroauricular mass associated with hearing loss. Initially, it was managed as mastoiditis. It refers to the worsening of the lesion with a progressive increase in size until the involvement of the right temporal bone and cheekbone, with insufficient coverage and bone exposure. Patient reported that approximately the same evolution time presents edema, erythema, heat, local secretion and undercoverage at the level of the first and fifth toe of the left foot with violaceous coloration of the fourth finger; she has a diagnosis of rheumatoid arthritis of 20 years of evolution in the treatment with prednisolone $10 \mathrm{mg}$, allergic to methotrexate and chloroquine. At the physical examination, patient presented multiple ulcerated lesions with irregular edges of necrotic background in the right temporal bone with destruction of the auricle, another extensive lesion in the right patella and at the level of the left foot knuckles. The articular exam shows a marked bone resorption of the distal phalanges of hands and feet associated with ulnar deviation and marked atrophy of the interosseous muscles, presents deformities in lower limbs associated with disability for displacement and the requirement to use a wheelchair. It was evaluated by the dermatologist who performed a biopsy of the temporal lesion. The skin fragments with representation of ulcer with fibrin and granulation tissue (newly formed capillaries and acute inflammation), in the underlying dermal stroma with a dense infiltrate of diffuse character. Neutrophilic with suppurative foci. There were some giant multinucleated cells (Figures 1-3).

Taking into account, the clinical findings and the antecedent of mutilans rheumatoid arthritis together with histopathological findings hitherto suggestive of pyoderma gangrenosum. Treatment indicated was systemic steroids at a dose of $1 \mathrm{mg} / \mathrm{kg} /$ day. Before starting steroids, in the context of an immunosuppressed patient an active search for tuberculosis began. Deworming was indicated. Subsequently, the biopsy report was made on January 24, 2017 for skin of the right ear. The lesion of the biopsy represent an ulcerative lesion with chronic inflammation and nonspecific character. Right skin of the atrial cover, actinic damage with chronic inflammation. Microscopically, fragment of soft tissues with representation of the epidermal surface by ulceration, deep cartilage, in the stroma. There was observed mixed inflammatory infiltrate with fibrin and acute inflammation. On the surface and more deeply neutrophilic without observing granulomas, no microorganisms were visualized either. No malignancy is observed. With this report, added to the patient record, dermatology considered pyoderma gangrenosum as the most likely diagnosis. After one month of treatment and with the persistence of the lesions, the treatment with ciclosporin was decided with a dose of $3 \mathrm{mg} / \mathrm{kg} /$ day with clinical improvement and re-epithelialization of the lesions (Figure 4 and 5).

\section{DISCUSSION}

There is a strong association between history of systemic diseases and the development of pyoderma

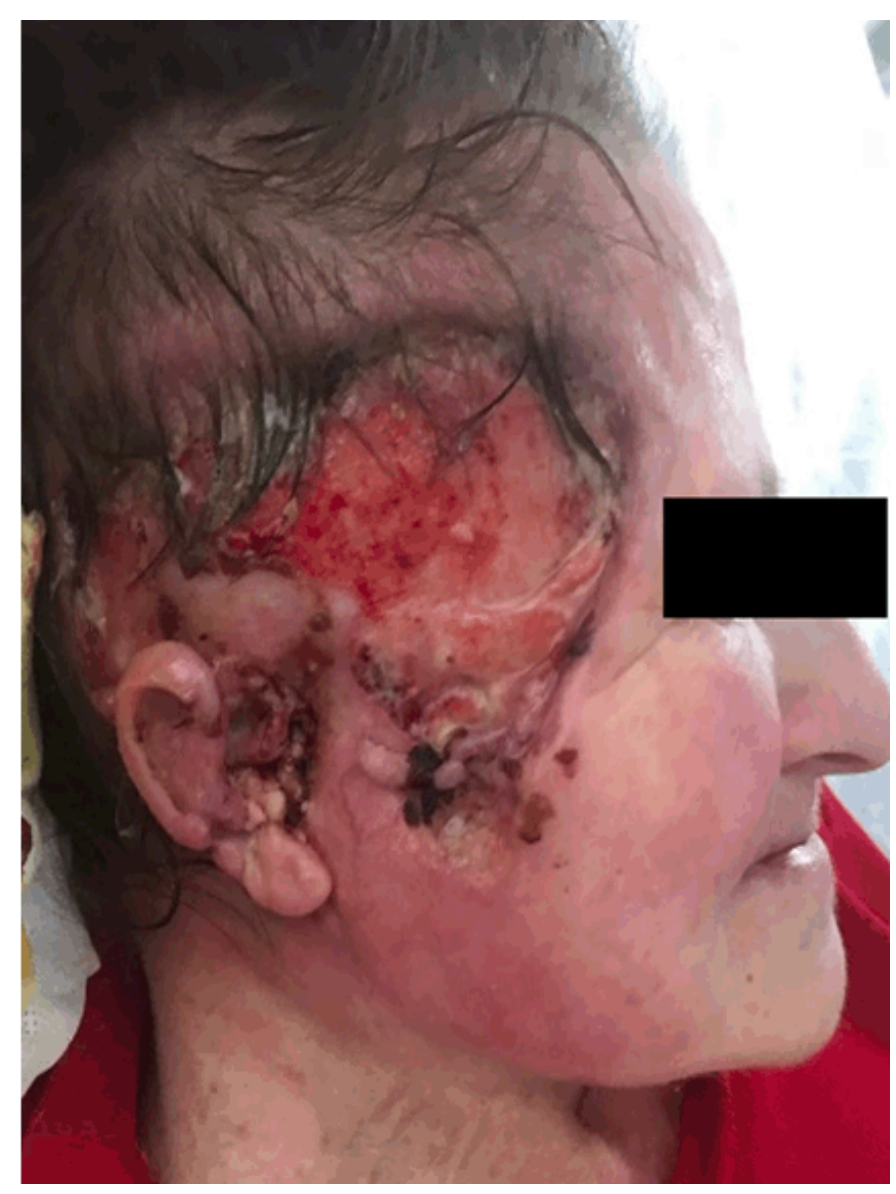

Figure 1: Right temporal lesion at the beginning of the treatment. 


\section{EDORiUM Journals}

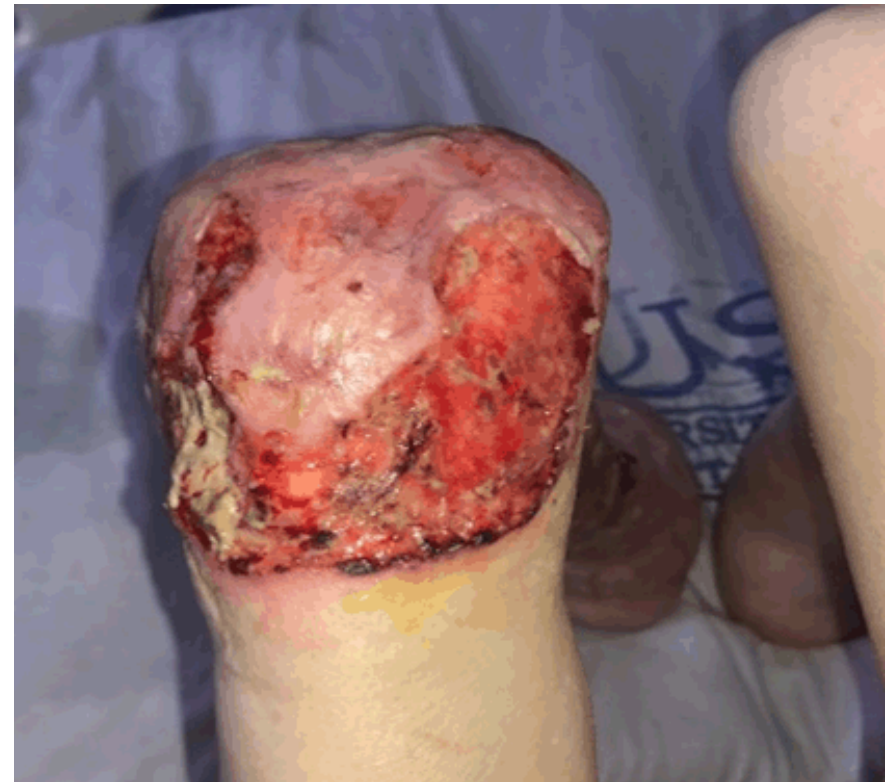

Figure 2: Right lower limb.

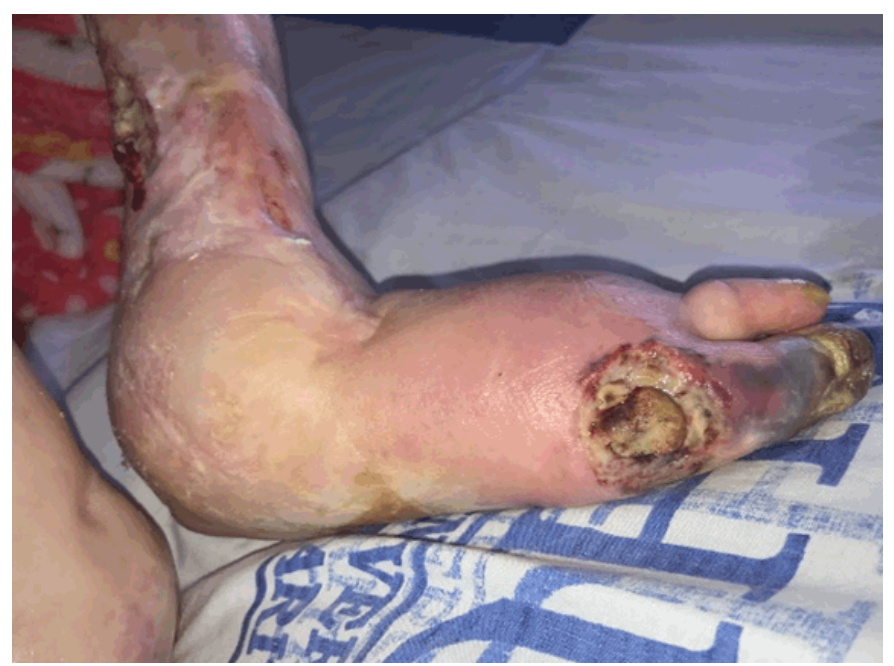

Figure 3: Left foot.

gangrenosum, some authors associate the pyoderma type with the corresponding systemic disease, finding that more than $70 \%$ of patients with ulcerative pyoderma gangrenosum have a systemic disease, such as inflammatory bowel disease, arthritis, monoclonal gammopathy and malignancy [8, 9]. On the other hand, it is also found that the phenomenon of pathergy develops in $25 \%$ of patients with the type of ulcerative pyoderma gangrenosum [9]. This supports the hypothesis that the patient in the case presented with an ulcerative variant pyoderma gangrenosum, since presented a phenomenon of pathergy at the beginning of the development of lesions, in addition to having a history of rheumatoid arthritis. However, skin lesions when a patient has rheumatoid arthritis are challenging, as they can include livedo reticularis, gangrene, vasculitis, purpura, and rheumatoid

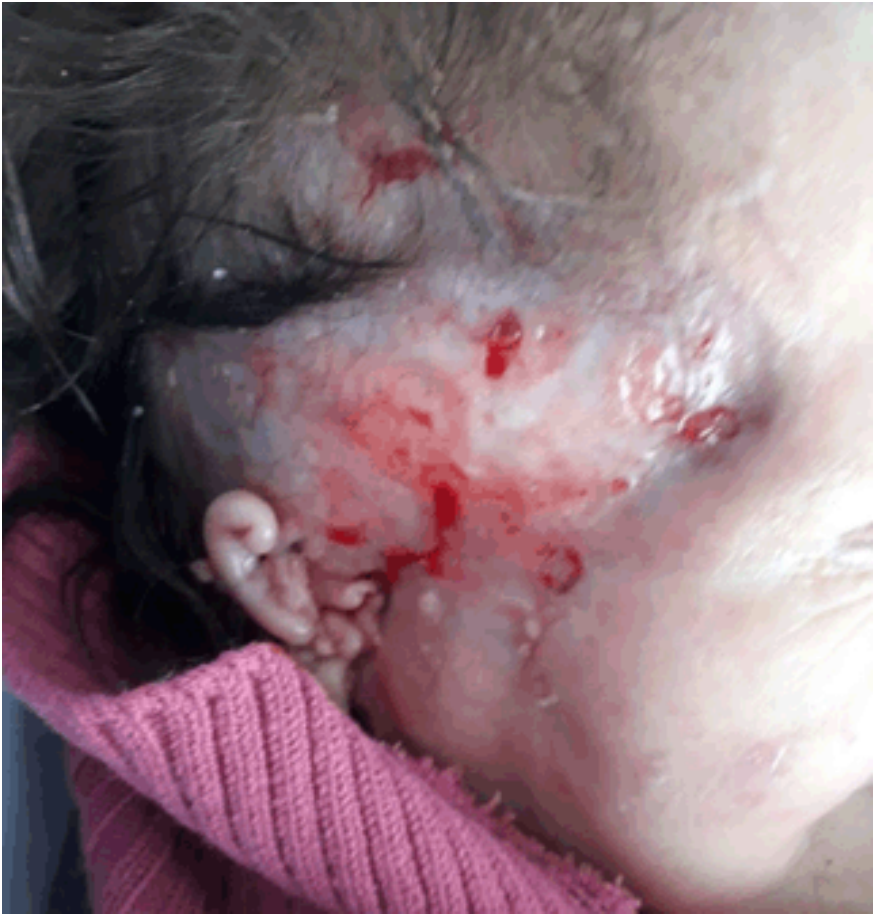

Figure 4: Post-treatment lesion.

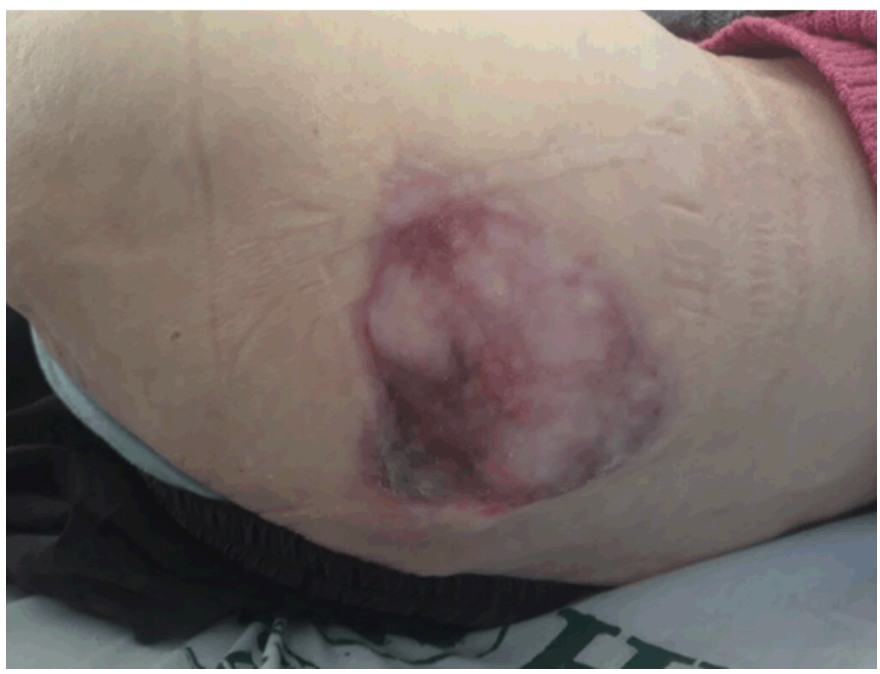

Figure 5: Right lower limb.

nodules $[10,11]$. These lesions occur predominantly in the lower limbs (78\%), trunk (11.7\%), upper limbs (8.7\%) and in the head and neck (7.8\%) [12, 13]. In this case, these lesions were presented in different locations with lower limbs as the most common, while head and neck as the least common location. It is important to highlight that one of the largest case series reported, found that only $7 \%$ had a biopsy suggestive of pyoderma gangrenosum, concluding in the same study that the biopsy of a lesion of PG rarely shows characteristics of the disease and should not be used to exclude diagnosis [10]. As noted in this series of cases is consistent with the results obtained in the patient's biopsy, which, although inconclusive, did 


\section{EDORiUM Journals}

not rule out the presence of pyoderma. In this regard and taking into account the above, the diagnosis of pyoderma gangrenosum was considered clinical, according to the findings and characteristics of the lesion (Currently), there is no serological support and the histopathology of each type of PG is not yet well defined, vascular immunofluorescence cast doubt on,but it is considered that in the future it could be useful in diagnostic support [9]. In this way, getting to identify that skin lesions are caused by PG disease is difficult and complicated, a differential diagnosis must be made with other entities [9]. In this case, one of the strongest differential diagnoses was Leishmaniasis, since first report of direct positive test for amastigotes was obtained, later another negative report. However, given the importance of leishmania in a Latin American population, where there are endemic sites for it with lesions of years of evolution, treatment was indicated without observing improvement of the lesions, nor epidemiological linkage. In addition to, a second negative report and subsequent biopsy that ruled out this diagnosis. The literature reports the use of different medications, both topical and systemic in patients with PG, without yet achieving a single management or protocol for these patients, this revels the complexity in the treatment of the disease. Much of the literature focuses on the use of systemic corticosteroids, such as prednisone at high doses, likewise the use of dapsone, minocycline, tacrolimus, cyclosporine and even the use of biological products, such as infliximab [12]. In our patient, different topical agents were used for the healing performed by wound clinic, in addition to the initiation of high doses of prednisone, as well as the later use of cyclosporine. It was from the use of this medication that improvement in head and face lesions was observed, however, refractoriness was seen in lower limb lesions.

The patient management with PG is complex and must always be supported by a multidisciplinary team [14]. This is how dermatology services, wound clinic, internal medicine, nursing, psychology, psychiatry and nutrition were included in different stages of diagnosis and treatment, in order to provide the best guarantees for the patient and to achieve the resolution of the lesions. It is necessary to mention, lesions caused by the PG are aggressive and with tissue damage, being able to persist for months or years, leaving scars in most cases. Although, there was a favorable evolution seen in the patient with a decrease in the lesions diameter and reepithelialization, it was impossible to obtain the appearance that the skin had before those lesions, this is due to the aggressiveness with which they appeared.

In recent years, surgical procedures have been considered in patients with PG, as an alternative in refractory cases, these procedures should always be accompanied by immunosuppressive therapy. In addition, the possibility that patients perform pathergy should also be considered [15]. In this case report, the patient improved the appearance of head and face ulcerative lesions. However, the lower limb lesions were refractory, which is why she required the amputation of the limb, without the development of pathergy nor later infections, and immunosuppressive therapy was continued after hospital discharge.

\section{CONCLUSION}

Pyoderma gangrenosum is an entity of difficult identification, diagnosis and treatment, with complications and changes in appearance that terribly affect the quality of life of those who suffer it. It is a challenge for all health personnel involved in patient care, so it is important to note that whenever a patient has a systemic disease base, as in this case rheumatoid arthritis, we should think of secondary entities to be associated, such as pyoderma gangrenosum; with adequate observation of the characteristics of each lesion and the request for diagnostic support, such as skin biopsy, keeping in mind the fact that the clinical characteristics of each patient have the greatest importance and that the biopsy is not exclusive in the case of pyoderma gangrenosum.

\section{REFERENCES}

1. Ruocco E, Sangiuliano S, Gravina AG, Miranda A, Nicoletti G. Pyoderma gangrenosum: An updated review. J Eur Acad Dermatol Venereol 2009 Sep;23(9):1008-17.

2. Wallach D, Vignon-Pennamen MD. Pyoderma gangrenosum and Sweet syndrome: The prototypic neutrophilic dermatoses. $\mathrm{Br} \quad \mathrm{J}$ Dermatol 2018 Mar;178(3):595-602.

3. Su WP, Davis MD, Weenig RH, Powell FC, Perry HO. Pyoderma gangrenosum: Clinicopathologic correlation and proposed diagnostic criteria. Int $\mathrm{J}$ Dermatol 2004 Nov;43(11):790-800.

4. Andreychik CM, Elston DM. Neutrophilic dermatoses. In: Hall JC, Hall BJ, editors. Cutaneous Drug Eruptions: Diagnosis, Histopathology and Therapy. London: Springer-Verlag; 2015. p. 375-87.

5. Chatzinasiou F, Polymeros D, Panagiotou M, Theodoropoulos K, Rigopoulos D. Generalized pyoderma gangrenosum associated with Ulcerative colitis: Successful treatment with infliximab and azathioprine. Acta Dermatovenerol Croat 2016 Apr;24(1):83-5.

6. Ahronowitz I, Harp J, Shinkai K. Etiology and management of pyoderma gangrenosum: A comprehensive review. Am J Clin Dermatol 2012 Jun 1;13(3):191-211.

7. Brooklyn T, Dunnill G, Probert C. Diagnosis and treatment of pyoderma gangrenosum. BMJ $2006 \mathrm{Jul}$ 22;333(7560):181-4.

8. Crowson AN, Mihm MC Jr, Magro C. Pyoderma gangrenosum: A review. J Cutan Pathol 2003 Feb;30(2):97-107.

9. Ahmadi S, Powell FC. Pyoderma gangrenosum: Uncommon presentations. Clin Dermatol 2005 NovDec;23(6):612-20. 
www.ijcasereportsandimages.com

10. Seitz CS, Berens N, Bröcker EB, Trautmann A. Leg ulceration in rheumatoid arthritis-an underreported multicausal complication with considerable morbidity: Analysis of thirty-six patients and review of the literature. Dermatology 2010;220(3):268-73.

11. Hasegawa M, Nagai Y, Sogabe Y, Clinical analysis of leg ulcers and gangrene in rheumatoid arthritis. J Dermatol 2013 Dec;40(12):949-54.

12. Binus AM, Qureshi AA, Li VW, Winterfield LS. Pyoderma gangrenosum: A retrospective review of patient characteristics, comorbidities and therapy in 103 patients. Br J Dermatol 2011 Dec;165(6):124450.

13. Kratzsch D, Ziemer M, Milkova L, Wagner JA, Simon JC, Kendler M. Facial pyoderma gangrenosum in senescence. Case Rep Dermatol 2013 Oct 11;5(3):295300.

14. Langan SM, Groves RW, Card TR, Gulliford MC. Incidence, mortality, and disease associations of pyoderma gangrenosum in the United Kingdom: A retrospective cohort study. J Invest Dermatol 2012 Sep;132(9):2166-70.

15. Khajehnoori M, O'Brien T. A case of surgically treated peristomal pyoderma gangrenosum in a patient with rheumatoid arthritis. J Surg Case Rep 2016 Jun 14;2016(6). pii: rjw103.

$* * * * * * * * *$

\section{Author Contributions}

Barros CE - Substantial contributions to conception and design, Acquisition of data, Analysis and interpretation of data, Drafting the article, Revising it critically for important intellectual content, Final approval of the version to be published
Concha DC - Substantial contributions to conception and design, Acquisition of data, Analysis and interpretation of data, Drafting the article, Revising it critically for important intellectual content, Final approval of the version to be published

Tapias SA - Substantial contributions to conception and design, Acquisition of data, Analysis and interpretation of data, Drafting the article, Revising it critically for important intellectual content, Final approval of the version to be published

\section{Guarantor of Submission}

The corresponding author is the guarantor of submission.

\section{Source of Support \\ None}

\section{Consent Statement}

Written informed consent was obtained from the patient for publication of this case report.

\section{Conflict of Interest}

Authors declare no conflict of interest.

\section{Copyright}

(C) 2018 Barros CE et al. This article is distributed under the terms of Creative Commons Attribution License which permits unrestricted use, distribution and reproduction in any medium provided the original author(s) and original publisher are properly credited. Please see the copyright policy on the journal website for more information.
Access full text article on other devices

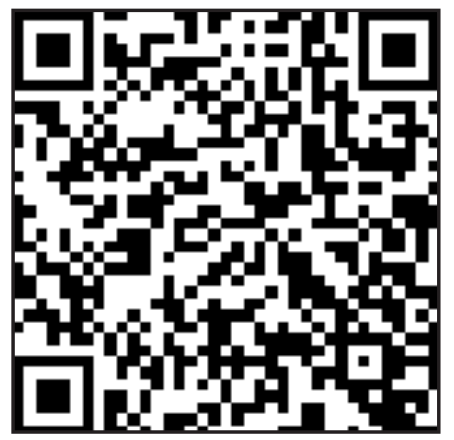

Access PDF of article on other devices

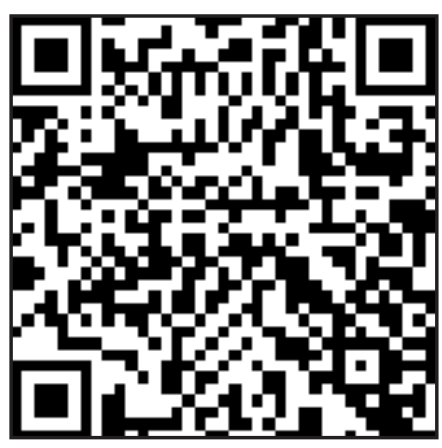

